\title{
Propuesta de un método de ontología crítica, composición entre genealogia y análisis estructural de contenido*
}

\author{
[Versión en Castellano] \\ Proposal for a Method of Critical Ontology, Composition Between \\ Genealogy and Structural Analysis of Content
}

Proposta de um método de ontologia critica, composição entre genealogia e análise estrutural de conteúdo

Recibido el 6 de mayo, 2019. Aceptado el 18 de diciembre, 2019.

Oscar-Armando Jaramillo-García**
https://orcid.org/0000-0002-6580-7847

Colombia

- Para citar este artículo:

Jaramillo-García, Oscar-Armando

(2020). Propuesta de un método de ontología crítica, composición entre genealogía y análisis estructural de contenido.

Ánfora, 27(49). 99-124.

https://doi.org/10.30854/anfv27.n49.2020.740 Universidad Autónoma de Manizales. ISSN 0121-6538/ e-ISSN 2248-6941. CC BY-NC-SA 4.0

\section{Resumen}

Objetivo: formular un método de investigación ensamblado para el análisis de procesos de subjetivación insertos en relaciones de poder-saber, a partir de las prácticas y gramáticas sociales que los constituyen. Desde allí, se busca una analítica que lea estas relaciones en investigaciones transversales sobre

* El presente artículo surge de la reflexión metodológica de la tesis doctoral: Prácticas artísticas y procesos de subjetivación en experiencias de la música y la danza en la ciudad de Pereira (Colombia), realizada entre los años 2013 y 2018 en el doctorado en Ciencias Sociales, Niñez y Juventud, en el marco del convenio CINDEUniversidad de Manizales. El autor declara que no hubo conflictos de intereses en la ejecución del proyecto de investigación.

** Doctor en Ciencias Sociales, Niñezy Juventud, Universidad de Manizales-CINDE. Estudiante Posdoctorado en Investigación en Ciencias Sociales CLACSO-CINDE. Docente en las Universidades: Fundación Universitaria del Área Andina (Pereira, Colombia), Universidad Tecnológica de Pereira y Universidad de Manizales. Correo electrónico: oscar.jaramillo@utp.edu.co 
el aquí y el ahora, para emplear en los discursos y subjetividades contemporáneos. Metodología: se aplicó una composición metodológica entre claves categoriales de la caja de herramientas foucaultiana, dos categorías articuladoras de la propia cosecha y técnicas de tratamiento de datos del análisis estructural de contenido. Resultados: se despliegan claves categoriales retomadas y otras desarrolladas a partir de lecturas foucaultianas y deleuzianas. Se ofrece una argumentación epistemológica para indicar el sentido del ensamblaje como lugar y función que cumple cada una de las fuentes metodológicas en medio de esta composición. Finalmente, se señalan algunos principios de descripción y estructuras simbólicas para el análisis de los datos. Conclusiones: este despliegue permite dejar sobre el escenario una elaboración metodológica, la cual aporta a los abordajes de las prácticas sociales y de subjetivación insertas en medio de relaciones de saber-poder, con lo que se propicia una vía para usar la caja de herramientas foucaultianas, que en Colombia ha sido tradicionalmente de trabajo con archivo; se logra una ruta para abordar discursos vivos desde una analítica gubernamental transversal.

Palabras-clave: Ontología crítica; Genealogía; Análisis estructural de contenido; Composición de métodos.

\begin{abstract}
Objective: to formulate a research method assembled for the analysis of subjectivation processes inserted in power-knowledge relationships, based on the social practices and grammars that constitute them. From there, an analyst is sought in order to read these relationships in cross-sectional research on the here and now, to be used in contemporary discourses and subjectivities. Methodology: a methodological composition was applied between categorical keys of the Foucauldian toolbox, two articulating categories of the harvest itself and data processing techniques of the structural analysis of content. Results: retained categorical keys and others developed from Foucauldian and Deleuzian readings are displayed. An epistemological argumentation is offered to indicate the sense of assembly as a place and function that each of the methodological sources fulfills in the middle of this composition. Finally, some principles of description and symbolic structures for data analysis are indicated. Conclusions: this deployment allows to establish a methodological elaboration, which contributes to the approaches of social practices and subjectivation inserted in the midst of knowledge-power relations, which provides a way to use the Foucauldian toolbox, which in Colombia has traditionally consisted of working with archives; a route to address live speeches from a transversal governmental analysis is achieved.
\end{abstract}


Keywords: Critical ontology; Genealogy; Structural analysis of content; Method composition.

\section{Resumo}

Objetivo: formular um método de pesquisa montado para a análise de processos de subjetivação inseridos em relações de poder-saber, com base nas práticas e gramáticas sociais que os constituem. A partir daí, busca-se uma análise que leia essas relações em pesquisas transversais do aqui e agora, para serem usadas nos discursos e subjetividades contemporâneos. Metodologia: foi aplicada uma composição metodológica entre chaves categóricas da caixa de ferramentas Foucaultiana, duas categorias articuladas da própria colheita e técnicas de processamento de dados da análise estrutural de conteúdo. Resultados: chaves categóricas retidas e outras desenvolvidas a partir de leituras foucaultianas e deleuzianas são exibidas. Uma argumentação epistemológica é oferecida para indicar o sentido de reunião como um lugar e uma função que cada uma das fontes metodológicas cumpre no meio dessa composição. Por fim, são indicados alguns princípios de descrição e estruturas simbólicas para análise dos dados. Conclusões: esse desdobramento permite deixar em cena uma elaboração metodológica, que contribui para as abordagens das práticas sociais e da subjetivação inseridas no meio das relações conhecimento-poder, que possibilitam a utilização do caixa Ferramentas foucaultianas, que na Colômbia tradicionalmente trabalham com arquivos; é alcançada uma rota para abordar discursos ao vivo a partir de uma análise transversal do governo.

Palavras-chave: Ontologia crítica; Genealogia; Análise estrutural de conteúdo; composição de Mêtodos. 


\section{Introducción}

En este artículo se elabora un diseño metodológico desde un enfoque post-estructuralista, fundamentalmente foucaultiano, pertinente para trabajar con objetos de estudio que aborden prácticas sociales y procesos de subjetivación. En este sentido, se entiende que es la obra de Foucault la posibilidad de contar con una caja de herramientas categorial como analítica, desde una apuesta histórico-filosófica de fragmentos filosóficos en talleres históricos (Leonard, 1985). Foucault no era un hombre obsesionado por el método, más bien lo contrario. Así lo afirmaba él (citado por Trombadori, 2010):

Puesto que nunca sé cuándo comienza un trabajo, qué pensaré al concluirlo, me resulta difícil señalar con claridad qué método empleo. (...) Una vez terminado el trabajo, ciertamente puedo, de una manera más o menos retrospectiva, extraer una reflexión metodológica a partir de esa experiencia. (...) Por lo tanto, yo no construyo un método general definitivamente válido para mí o para otros (p. 43).

En consecuencia, se puede afirmar que no hay un método consuetudinario u obligatorio para investigar desde la óptica foucaultiana; es más, se han generado diversas vías metodológicas desde la caja de herramientas del francés. Así pues, en este texto interesa seguir la estela que ha dejado un grupo de investigación que se crea en Colombia desde varias universidades e investigadores; este se nombró y aún se conoce como el Grupo de Historia de la Práctica Pedagógica.

Este grupo introdujo, desde mediados de la década del setenta, el uso de pistas foucaultianas para dar vida a un diseño metodológico propio del grupo, con marcas claras, aunque se pueden ver algunas particularidades en el ejercicio de cada investigador. Allí aparece entonces la arqueología y luego la arqueología-genealogía como posibilidad metodológica para la investigación. Hay que decir que la apuesta de estos investigadores busca seguir muy de cerca las indagaciones foucaultianas; así, se dedican a unos abordajes históricos en el lugar del archivo, con la finalidad de hallar el campo de dispersión enunciativo y los enunciados que emergen en diferentes cortes históricos.

Hay que anotar que la mayoría de los investigadores del grupo se han mantenido en la línea original de la arqueología y la arqueología-genealogía; tal vez han sido Oscar Saldarriaga (2008) y Javier Sáenz (2014), quienes han propuesto algunas variantes. El primero, ligando las pistas de análisis foucaultiano al método de análisis estructural de contenido (MAEC) en la línea de Hiernaux (2008), pero sin salir del espacio de la investigación historiográfica de archivo. El segundo, desde no hace más de ocho años viene realizando aportes para una grilla de análisis, más no un método, que permita leer las prácticas de sí contemporáneas, saliendo del espacio del archivo y llevando la analítica al ámbito de las fuentes primarias. 
En este sentido, esta propuesta quiere seguir generando aportes al campo metodológico que se inquieta por las relaciones de poder, la gubernamentalidad y los procesos de subjetivación, marcando una línea que articula claves foucaultianas con el MAEC, para el trabajo localizado con subjetividades y discursos vivos. En consecuencia, el aporte central de este trabajo, en el escenario académico colombiano, es que logra un movimiento de las apuestas tradicionales arco-genealógicas y se desplaza a un dominio más allá del archivo. Es decir, usa algunos de los elementos de la caja analítica foucaultiana potenciándola con otras categorías y técnicas de análisis, lo que otorga la posibilidad de realizar un trabajo con participantes específicos que formulan su discurso en el aquí y ahora, lo que hace pasar del archivo a las fuentes primarias. Ello, desde un ejercicio de diseño argumental y lógico que busca mantener la coherencia epistemológica y procedimental que reclama un método de investigación pertinente.

\section{Un método en clave de ontología crítica}

Ahora bien, se pone de relieve que la presente propuesta de método se inscribe en el proyecto foucaultiano de una ontología crítica de nosotros mismos, en línea de los estudios gubernamentales que abordan las sociedades de control. En este sentido, la apuesta está en el lugar de las preguntas por ese presente que somos, la manera en que estamos siendo formados-gobernados, nos formamos-gobernamos (Sáenz, 2014). En esta medida puede decirse con Foucault que:

La ontología crítica de nosotros mismos (...) hay que concebirla como una actitud, un ethos, una vida filosófica en la que la crítica de lo que somos es a la vez análisis histórico de los límites que nos son impuestos y prueba de su posible transgresión. (Foucault, 2003c, p. 97).

Entonces, investigar desde un método ligado a la labor de ontología crítica del presente lleva a una problematización de los modos en que hemos devenido subjetividad, poniendo en juego su dominio, para reconocer las racionalidades y dispositivos que han permitido crear ciertas experiencias de sí. Por lo tanto, buscando entender qué hace que hoy nos nombremos como nos nombramos, asumamos las posiciones de sujetos unidimensionales, sólidas o múltiples, que tomamos, hay que reconocer las condiciones de posibilidad en su honda densidad histórica, discontinua y contingente, para poder observar las emergencias de las líneas de fuerza que van mostrando nuevas configuraciones en marcha.

Por consiguiente, una indagación ontológica no se pregunta ¿̇qué es el ser? o ¿qque es el sujeto?; es antiesencialista y nominalista, partiendo de un escepticismo 
metódico y sistemático ante todo universal antropológico (Foucault, 1999b). La ontología traza más bien la ruta de una inquietud por un ¿̇cómo se constituye la subjetividad?, explicitando así que esta ontología no es sustancialista, no es trascendental, fundamentada en leyes o categorías transhistóricas o metafísicas. A este tenor, un sujeto que emerge de prácticas históricas, y que por tanto es contingente, no es el mismo de una vez y para siempre; por eso, es pertinente preguntar por su posición subjetiva actual.

Así, un método para el análisis de los modos de subjetivación contemporáneos requiere ubicar las prácticas, como maneras complejas y múltiples de una gubernamentalidad que supone "por un lado formas racionales, procedimientos técnicos e instrumentaciones por medio de las cuales se ejerce y, por otro, juegos estratégicos que tornan inestables y reversibles las relaciones de poder que aquellas deben asegurar" (Foucault, 2013, p. 192). Esto hace que en las artes de gobierno contemporáneas los procesos de subjetivación se muevan en medio de tensiones y formas ambivalentes; no es la subjetividad una forma clara e inmóvil. Es decir, las fuerzas y formas (Deleuze, 2014) en medio de las que cobra vida la subjetividad, pueden contribuir tanto a potenciar el gobierno de unos hombres sobre otros, como al gobierno de un sujeto sobre sí mismo.

Por tanto, valga recalcar, para entender el funcionamiento de estas prácticas, que no operan en el vacío o como un algo independiente, en lo que es importante hacer alusión a la categoría de focos de experiencia: “donde se articulan unos con otros: primero, las formas de un saber posible; segundo, las matrices normativas de comportamiento para los individuos, y por último, modos de existencia virtuales para sujetos posibles" (Foucault, 2009, p. 19).

Estos tres elementos permiten señalar que las prácticas no pueden pensarse como un tipo de práctica que únicamente convoca a la subjetividad, entendida en términos solipsistas, categorial y analíticamente hablando. Las formas de pensar, decir, sentir, hacer y conducirse del sujeto pueden asumirse desde Foucault en tres espacios interconectados: campos de saber que le permiten a los sujetos concebirse en términos de muy variados discursos y la positividad de sus verdades, pudiendo ser estos sociales, jurídicos o humanistas por nombrar algunos; las relaciones estratégicas de poder que ligadas a enunciaciones dan paso a prácticas no-discursivas que quieren determinar las acciones que el sujeto lleva a cabo; y el trabajo ético del sujeto sobre sí mismo que le permite constituir formas de subjetividad que resistan a las relaciones de poder que quieren determinarle sin su injerencia crítica o de otro lado, trabajo que le sirve para que afirme su lugar, pero desde una posición reflexiva y deliberada.

En consecuencia, un método que se inscribe en la reflexión de la ontología crítica foucaultiana, tiene la capacidad para indagar por el presente que somos y el modo en que estamos siendo constituidos. Por tal razón, se despliega en este 
artículo un ensamblaje que pueda hacer inteligibles, desde la lectura de los discursos y decires de las subjetividades contemporáneas, los modos de gobierno de la vida que son característicos del capitalismo neoliberal globalizado.

\section{Metodología}

En la actualidad el ámbito de lo metodológico, entendido desde la problematización del enfoque cualitativo (Creswell, 2007; Hernández-Sampieri, Fernández y Baptista, 2010), se muestra como muy dinámico pues se cuenta con una gran cantidad de posibilidades para abordar los objetos de investigación. Así, no es una novedad la composición de métodos (Herrera, 2010, 2013) que se generan al interior del enfoque cualitativo, que refiere la articulación o integración de dos o más métodos. No obstante a la regularidad de estas composiciones, su problema en ocasiones radica en la falta de rigor epistemológico, lo que puede terminar en contradicciones en medio de la lógica de producción de conocimiento que moviliza cada método, la concepción de realidad, el devenir histórico, la comprensión de sujeto, verdad o la posición política en que se ubican (Jaramillo, 2013). En ello, si ciertos diálogos no son justificados pueden terminar en medio de la confusión, traslape o la dilución de aquellos elementos de las realidades sociales que un método u otro se interesa en comprender o de otro lado critican y no asumen.

De este modo, se propone un diseño de método compuesto, que permita un abordaje analítico en clave de ontología crítica (Foucault, 2003c), que vaya como unidad de análisis sobre las prácticas sociales (Veyne, 1984; Castro-Gómez, 2010) para visibilizar procesos de subjetivación (Jaramillo, 2013). De esta suerte, se postula un diálogo entre líneas fuerza de algunas claves metodológicas foucaultianas (Foucault, 1985, 1994, 2002, 2003a, 2003b, 2006a, 2006b, 2008, 2009), fundamentalmente las llamadas genealógicas, dos categorías de la propia autoría y el MAEC que emerge desde la propuesta e influencias de Hiernaux (Hiernaux y Ganty, 1977; Hiernaux, 2008; Suárez, 2008; Saldarriaga, 2003, 2008). De tal manera, el posicionamiento epistemológico implica que el MAEC es territorializado desde un enfoque postestructuralista, pues las claves de ontología crítica se complementan con grafos o matrices de análisis y dimensiones específicas, que permiten visibilizar la complejidad de las prácticas en la producción de subjetividades desde una mirada de las relaciones de saber-poder y no desde un punto vista psicológico o sociológico. 


\section{Resultados}

\section{Claves analíticas: el juego diagramas de poder, gramáticas sociales y gramáticas de vida}

Se reafirma que las prácticas son centrales para el análisis dentro de esta composición de método, comprendiendo que "la práctica no es una instancia misteriosa, un subsuelo de la historia, ni un motor oculto: es lo que hacen las personas (como lo indica la propia palabra)” (Veyne, 1984, p. 207): unas prácticas que se generan en un espacio de reglas particular, en un campo de interacciones posibles que se podría nombrar como lo hace Veyne, la gramática de las prácticas. De esta forma, el gobierno de unos hombres sobre otros y el gobierno de un sujeto sobre sí mismo, no existen por fuera las prácticas. Entonces, un análisis de las prácticas implica reconocerlas como el punto de partida del análisis: "Las cosas, los objetos no son más que el fruto de las prácticas (...) En efecto, ’los gobernados' no son ni una entidad única ni múltiple (...) sólo existen múltiples objetivaciones que corresponden a prácticas heterogéneas” (Veyne, 1984, p. 215).

Hay numerosas objetivaciones, y eso es todo. La relación de esa multiplicidad de prácticas con una unidad no se plantea más que si se intenta darles una unidad que no existe. Bajo estos preceptos no hay algo por debajo de las prácticas que debe ser hallado; en ellas aparece, como producción socio-histórica, su propio régimen aglutinador, su gramática, su propia racionalidad, como una racionalidad entre otras racionalidades. En esta línea, las prácticas se entienden como el lugar en el cual se debe iniciar el análisis; no se parte entonces de una concepción definida y delimitada por conceptos humanísticos sobre la subjetividad, más bien se deja traslucir que son las prácticas las que dan vida a modos particulares de subjetivación que requieren ser entendidos de manera situada según sus condiciones históricas de posibilidad (Muñoz y Jaramillo, 2019; Jaramillo, 2018).

De esta manera, el análisis no es de enunciados en términos arqueológicos; se rompe con la búsqueda de campos de dispersión enunciativa y en esa forma con la unicidad del archivo documental, apostando por un andamiaje metódico que le dé cabida a la heterogeneidad de las fuentes. De esta suerte, si bien se quiere ir más allá del archivo como lugar total de análisis, no se desconoce la existencia de campos de saber, más bien el interés está en recodificarlos con otras posibilidades discursivas y articular así con lo no estratificado ${ }^{1}$ (Deleuze, 1987, 2014).

Por ello, las relaciones de poder, el espacio de las fuerzas que afectan y son afectadas y que se entiende desde su especificidad relacional e histórica como 
diagrama (Deleuze, 2014) quieren ensamblarse con dos categorías de la propia cosecha de quien escribe el artículo: el doble juego de las gramáticas sociales y las gramáticas de vida² que son útiles para pensar desde una análitica de lo contemporáneo los procesos de subjetivación. En primera instancia, hay que reafirmar las relaciones de poder desde una mirada gubernamental, donde se postula que "vivir en sociedad es vivir de modo tal que es posible actuar sobre la acción los unos de los otros. Una sociedad "sin relaciones de poder" sólo puede ser una abstracción” (Foucault, 1988, p. 255).

En consecuencia, puede decirse con Foucault que el poder está en todos lados, viene de todas partes (2002); desde esta óptica no se conciben las relaciones humanas en sus diferentes dimensiones como fuera de las relaciones de poder. Consiguientemente, el poder es relación de fuerzas, una fuerza que tiene por objeto otra fuerza; las fuerzas son el elemento de una multiplicidad, son una acción sobre una acción o si se quiere en plural, acciones sobre acciones. En ello el poder actúa en la medida en que "incita, induce, disuade, facilita o dificulta, amplia o limita, vuelve más o menos probable” (Foucault, 1988, p. 238). Se trata de acciones de poder donde una fuerza manda y otra obedece (Deleuze, 2014) en una relación compleja, estratégica y reversible.

Ahora, las fuerzas según lo plantea Deleuze en su lectura de Foucault, se entienden formando un campo de relaciones que puede codificarse bajo el concepto de diagrama (Deleuze, 2014). Este se concibe como una situación estratégica compleja - múltiple - relacionada con el espacio y el tiempo. Por ejemplo, y sin ser los únicos posibles, aquella situación que se configura en un espacio-tiempo de encierro con una multiplicidad poco numerosa, o de gestión de la vida en una multiplicidad numerosa y en un espacio abierto. De este modo, toda formación social remite a un diagrama de poder que no es igual al de otra formación social; tiene así un carácter fluido, fluctuante y en esta medida siempre inestable; no hay equilibrio de la relación de fuerzas; el diagrama es entonces un lugar de mutación (Deleuze, 2014).

Se considera el diagrama como campo de fuerzas, no como estructura, sino como "una actividad de estructuración que atraviesa todas las estructuras esta-

2. Estas dos categorías, de la propia autoría, tienen como finalidad ser el lugar de articulación coherente entre las claves analíticas foucaultianas y el AEC. En este sentido, dichas categorías reemplazan el concepto de institución cultural, propuesto por Hiernaux, que se afinca en la psicología social y la fenomenología y al mismo tiempo se ponen en un lugar analítico diferente del análisis histórico de enunciados, propio de la arqueología foucaultiana, emergiendo como la posibilidad de llevar la caja de herramientas de Foucault al campo de trabajo con fuentes primarias en investigaciones transversales. De esta manera, logran ser un bloque de articulación entre una marco analítico que comprende el mundo desde procesos de subjetivación, marcados por prácticas discursivas y no discursivas, en medio de las cuales transitan relaciones de poder y unas potentes técnicas de análisis de datos como las del AEC, lugar donde está el sentido y la novedad de esta propuesta. 
bles que le corresponden, con una pluralidad de estructuras eventuales posibles" (Deleuze, 2014, p. 80). De esta suerte, las relaciones de fuerza están en perpetua mutación, por lo cual es difícil hablar de sociedad actual, pues ella es la conjunción de lo que está todavía desapareciendo y de lo que apenas está naciendo. "Ahora, hay que afirmar claramente que el diagrama está primero que las formas, que las formas derivan de él” (Deleuze, 2014, p. 111). En esta medida, las fuerzas que afectan y son afectadas - afectos activos y afectos reactivos - en el diagrama, permiten manifestar que este es: "abstracto sin ser general, diría que es virtual sin ser irreal, es virtual sin ser ficticio (...) Porque está hecho de pequeñas emergencias, micro-emergencias, de pequeñas emergencias y desvanecimientos" (Deleuze, 2014, p. 116).

Este punto indica que no hay diagramas estáticos; el diagrama es lo que está siendo; sus flujos hacen que no sea posible pensar una dominación perpetua, una obediencia indefinida y una resistencia definitiva y absoluta. Las fuerzas siempre están en medio de sus juegos, afectaciones, tensiones y flujos; pretenden modificar de uno y otro lado, con diferentes estrategias y en diferentes tiempos, el medio que habitan y la posición que allí asumen. En esto, el poder es la emisión de singularidades, la distribución de singularidades, luego este poder se actualiza en el saber, donde al tiempo se integra y se diferencia; en este sentido se formaliza en sustancias y en funciones (Deleuze, 2014). De esta manera, si hubiese que dar un nombre a las relaciones de poder, las relaciones de fuerza, se podría invocar a Foucault cuando sostiene que "el gobierno expresa las relaciones moleculares que constituyen el poder” (Foucault, citado por Deleuze, 2014, p. 119).

Siguiendo este argumento, las relaciones de fuerza difusas, las singularidades múltiples se actualizan en lo estratificado en elementos molares, y, cuando lo hacen, empiezan a operar en razón a una estrategia definida por una racionalidad dominante. Emerge la relación de poder-saber entendida como relación entre fuerzas y formas; fuerzas que se formalizan y que buscan conducir el campo de acción en que se mueve el otro, en razón a ciertos fines. Dan paso a un medio artificial de gramáticas que opera a partir de la relación entre diagramas de poder, campos de saber y relaciones de sí consigo.

De modo consiguiente, para lograr esta lectura de manera más precisa, hay que afirmar que en lo estratificado se actualiza la virtualidad de las fuerzas, integrándose y diferenciándose en funciones formalizadas y materias-formadas, pues si las fuerzas no están estratificadas, formadas, operan como imponer una tarea cualquiera a una multiplicidad humana cualquiera y los estratos permiten otros niveles de inteligibilidad pues "la función formalizada es una acción remitida a sus fines, sus medios, a los obstáculos que encuentra, etc.” (Deleuze, 2014, p. 75).

En este sentido, educar, corregir, hacer trabajar, curar, gobernar sobre otros, son funciones formalizadas que uno podría también girar a otro plano de guber- 
namentalidad refiriéndolas como educar-se, corregir-se, trabajar-se, curar-se, gobernar-se. Acciones que, ensambladas en medio de formaciones estratificadas concretas, son lo que Foucault llama dispositivos (Deleuze, 2014), punto donde se integran y diferencian, allí se codifican las fuerzas, han devenido estratificadas y diferenciadas dando lugar con ello al prisionero, el obrero, el estudiante, el joven, el artista, entre otros. Puntos estos centrales para un trabajo analítico pues permiten que la gubernamentalidad, en tanto relación poder-saber-verdad, pueda ser leída en el lugar del estrato donde tienen cabida las líneas del ver y el hablar (Deleuze, 2014).

Luego bien, ello permite decir que no hay poder sin saber ni saber sin poder, y que por esto el espacio del saber que actualiza las fuerzas es fundamental; es lo que permite llegar a los dos niveles de gramáticas que se quieren desplegar para hacer alusión finalmente al orden aglutinador de las prácticas. En el momento que diagrama y campos de saber se piensan en conjunción puede hablarse de las condiciones de posibilidad históricas que dan pie a la emergencia de:

Los códigos fundamentales de una cultura - los que rigen su lenguaje, sus esquemas perceptivos, sus cambios, sus técnicas, sus valores, la jerarquía de sus prácticas fijan de antemano para cada hombre los órdenes empíricos con los cuales tendrá algo que ver y dentro de los que se reconocerá (Foucault, 1981, p. 5).

Este espacio de saber se quiere acercar por lo tanto al primer nivel de gramáticas (Veyne, 1984; Castro-Gómez, 2010), las gramáticas sociales, que no son más que los códigos, entendidos como sistemas de reglas que hacen que lo múltiple sea posible en un momento histórico particular. Lugar donde se comprende lo múltiple como las diferentes posibilidades que tienen sociedades y sujetos de estructurarse y constituirse bajo regularidades que permitan dar forma a campos y juegos con reglas contingentes, pero de alguna duración.

Por eso, entender las gramáticas sociales en medio de los campos de saber es asumir que estas implican también: "Esa película de pensamiento implícito en las culturas que articula hasta los dominios más ínfimos de su modo de vida (...) el que hace posible, en un momento dado, la aparición de una teoría, de una opinión, de una práctica” (Morey, 1983, pp. 19-20). Así pues, estas gramáticas sociales son el campo de juego disponible, el lugar amplio en que la subjetividad es producida y en ello gobernada, al tiempo que también es el espacio social donde puede tomar posiciones y buscar sus lugares de enunciación y de funcionamiento.

Luego, este campo disponible por su amplitud y variabilidad de segmentos no puede ser tomado en pleno por la subjetividad, pero es el espacio en el que esta puede ir actualizándose, estando en una suerte de afuera (Deleuze, 2014) la posibilidad siempre latente de lo otro, de encontrar líneas de fuga, rutas para 
el nomadismo, fuerzas que operen como resistencia. O, de otra parte, formas normalizadoras del adentro, las siempre inminentes formas de gobierno de unos hombres sobre otros en estos espacios gramaticales, que hoy se toman inscritos en las racionalidades y tecnologías gubernamentales que atraviesan las sociedades de control (Deleuze, 1995).

De modo subsiguiente, se propone un segundo nivel gramatical que se encuentra ya no en lo disponible, sino que es aquello que se va haciendo subjetividad. Las gramáticas de vida son un nivel que se despliega a partir de las gramáticas sociales; este nuevo nivel no es el de las reglas sociales amplias y diversas, son más bien las reglas que han venido directamente a afectar la subjetividad desde el gobierno de unos hombres sobre otros, o también aquellas maneras que desde las prácticas transformadoras o auto-transformadoras se apropian para sí las subjetividades; son lo que efectivamente se está siendo, las que constituyen directamente la subjetividad.

En este orden, las gramáticas de vida pueden ser virtualmente elementos que, en los pliegues, despliegues y posicionalidad de la subjetividad, no poseen necesariamente una total coherencia y pueden aplicar de manera diferencial, pero a pesar de ello mantienen una cierta estabilidad en las prácticas. No obstante, este tipo de composición, las gramáticas de vida son los elementos de poder-saber en los que se producen-constituyen los trayectos subjetivos. De este modo, la subjetividad se configura/es configurada en forma/formas a partir de las prácticas, es decir, de aquello que piensa, siente, dice y hace, en tanto que piensa, siente, dice y hace.

Estas formas toman cuerpo a partir de las fuerzas y las funciones formalizadas que han hecho subjetivación, y en tanto se han traducido en prácticas regulares y lugares que permiten hacer inteligible esa posibilidad de ser en el mundo. Luego estas formas, que se configuraron en las redes de relaciones de fuerza y de discursos, son móviles, contingentes y pueden ser modificadas a partir de nuevos trayectos o nuevas interpretaciones de trayectos ya vividos, modificando así la inteligibilidad de cierto orden de prácticas.

\section{Territorialización: un análisis estructural de contenido codificado des- de el post-estructuralismo}

La lógica analítica que se plantea para el abordaje y tratamiento de los datos en esta composición es una que se ubica, con algunos matices, desde el ámbito del MAEC. Así, un instrumento metodológico que se inscribe en la sociología de la cultura y permite la descripción estructural y análisis de datos empíricos. Su intención de base es extraer de materiales concretos "las estructuras simbólicas de determinados actores sociales. La pregunta que pretende responder es cómo 
se constituye el sentido en materiales concretos” (Suárez, 2008, p.119). En razón a ello y en primera instancia, se quiere defender en términos epistemológicos el lugar de este tipo de análisis, en medio de un diseño que tiene sus topos de enunciación en el post-estructuralismo.

De tal manera, el post-estructuralismo emerge como un lugar de visibilidad fuerte, desde la década de los setenta, en cabeza de autores franceses como Foucault, Derrida, Deleuze, Guattari y Bourdieu, entre otros. Inicialmente, una característica central en esta mirada es la historización, con la cual se deja de lado la historia global, aquella que quiere restituir la forma de conjunto de una civilización, las leyes o las significaciones comunes a todos los fenómenos de un período (Foucault, 1976), en lo que se apuesta por una historia general, entendiendo relaciones, pero en medio de juegos de series y de espacios de dispersión (Foucault, 1976) que serán discontinuos, no finalistas, no evolucionistas. Mirada que muestra unas formas diferenciadas no universales, y sí locales, producidas en sus relaciones particulares de poder-saber, donde lo que es no ha sido siempre y ha aparecido en medio de la confluencia de reencuentros, de casualidades, al hilo de una historia frágil y precaria, que se marca por racionalidades estratégicas, por fuerzas (Foucault, 1999a).

No solo la historización y la introducción de la categoría de poder son elementos que remarcan el post-estructuralismo, también se han ocupado de la ética, la estética y la producción de subjetividades (Álvarez, 2014). En esta misma línea, y en medio de su escepticismo, han realizado una crítica desfundamentadora de la razón moderna, postulando diversas racionalidades donde "se dan diferentes instauraciones, diferentes creaciones y diferentes modificaciones por las cuales ciertas racionalidades se engendran unas a otras, se oponen unas a otras, se sustituyen unas a otras" (Foucault, 1999a, p. 318).

Otro punto que interesa resaltar es que el post-estructuralismo no va a eliminar al sujeto; empero, el nombrarlo no implica recuperar el sujeto moderno del ego cogito cartesiano o de la conciencia en términos fenomenológicos y humanistas. Es un tipo de sujeto que se entiende producido en medio de relaciones de gobierno que vehiculan saber-poder-verdad; así, no hay una esencia fundamental o naturaleza humana invariante, sino una producción histórica de subjetividades demarcadas por racionalidades estratégicas. Un sujeto que, a pesar de no estar por fuera de las relaciones de poder, puede tener algún grado de participación en su constitución por medio de prácticas de sí.

En consecuencia, y con un interés de clarificación epistemológica, se alude al topos de enunciación que asume el MAEC. Con esto, valga decir que es un análisis que en particular siguen algunos autores que, de diversas formas, han transitado por la escuela de la Universidad Católica de Lovaina la Nueva, como J. P. Hiernaux, Hugo José Suárez y Oscar Saldarriaga. En este sentido, estos plantean acerca del MAEC que: 
Sin ubicarse directamente en el linaje del estructuralismo clásico (...) retoma múltiples aportes fundamentales de autores de esta corriente, pero "colonizados" (...) a partir de un acercamiento propiamente sociológico que vincula la estructura con la cultura, con lo social y, en definitiva, con el sentido movilizador de la existencia de los actores sociales. (Suárez, 2008, p. 14).

Entonces, puede expresarse de manera clara que el análisis que aquí se realiza no se inscribe de manera tajante en estas fórmulas clásicas estructuralistas. Su aproximación a la sociología permite decir que se sale de la mirada que asume todo y únicamente en términos de relaciones de lenguaje sin subjetividades. Con lo cual, muestra la dinámica de los procesos sociales y culturales dando lugar a una producción de sentidos, que se instalan en medio de sistemas de reglas de combinación que movilizan estos lugares estructurales. Con ello, aparece el lugar de una estructura que no es invariable y, por ende, se puede asumir en medio de tramas históricas; en este caso desde la historia general, serial, múltiple y discontinua.

Al mismo tiempo, y con el fin de darle un mayor acento post-estructuralista al análisis, vinculando con potencia la gubernamentalidad y las relaciones de poder-saber, en medio de escenarios de producción de subjetividad operadados desde el gobierno de unos hombres sobre otros o desde el gobierno de sí, se pone en relación con los términos de gramática social y gramáticas de vida. A esta sazón, es central mostrar un desplazamiento conceptual que señala de manera categórica un mayor acercamiento a la manera en que se usa el MAEC en esta composición de método.

Así pues, Hiernaux plantea el concepto de institución cultural, producida e interiorizada socialmente, a la cual responde el ser humano como dotado de sentidos. En ello, tal institución cultural es un conjunto de "sistemas de reglas de combinación constitutivos de sentido, informando las percepciones, las prácticas y los modos de organización puestos en práctica por los actores” (Suárez, 2008. p. 15). Esta se va a dividir entre estructura cultural (lo estructural) y estructura social (lo estructurel); la primera alude al grupo de factores ligados a las condiciones subjetivas de la acción, es decir, aquello capaz de producir un sentido movilizador, así como una percepción del sí y de los otros estructurando, la percepción de lo normal y lo posible (Suárez, 2008). La segunda, la estructura social, hace alusión a las situaciones sociales en las cuales se encuentran inmersos los sujetos al contexto social e histórico particular. Se trata de las "condiciones objetivas” de la acción, es decir, los efectos de la estructura social propia en la cual el individuo se desenvuelve (Suárez, 2008).

Dichos conceptos, en este diseño, se reemplazan por el de gramáticas de vida y gramáticas sociales, que en alguna medida tienen ciertas similitudes. El primero se asemeja a la estructura cultural, pues las gramáticas de vida también 
aluden a los regímenes de prácticas que efectivamente cruzan al sujeto; y las segundas gramáticas tienen cierta similitud al de estructura social, pues estas aluden al sistema de reglas disponibles en que se estructura el conjunto de lo social.

Ahora, si bien hay cercanías, las gramáticas tienen un mayor énfasis post-estructuralista pues se asumen marcadas por diagramas, que aluden de forma clara y directa al lugar de las relaciones de poder, de fuerzas asimétricas que se producen en medio de las combinaciones gramaticales. En esta línea, estos juegos de reglas están atravesados por la gubernamentalización de la vida que fabrican las sociedades de control (Deleuze, 1995). Son también radicalmente históricas bajo una historicidad discontinua y múltiple que indica su contingencia y la lectura más allá de la racionalidad unidimensional. En la misma clave, las gramáticas son un escenario en el cual aparece la dinámica compleja de una subjetividad múltiple, que no es siempre igual a sí misma ni tampoco totalmente coherente o lógica, rompiendo con miradas que implican la psicologización del sujeto, sin recurrir a basamentos que marquen invariantes antropológicas, y la ubican en medio de la producción gubernamental de unos hombres sobre otros, pero también en el lugar de poder producirse a sí mismo, en algún grado de manera deliberada, por un trabajo de la subjetividad sobre sí misma (Foucault, 1999a).

Finalmente, en cuanto a este desplazamiento de talante post-estructuralista, valdría reseñar una última diferencia en el lugar del sentido. Se puede compartir con Hiernaux (2008) que existen unos sistemas de reglas de combinación constitutivos de sentido; no obstante, se toma distancia cuando los relaciona con representaciones y percepciones, propios de las psicologías sociales y la fenomenología. De este modo, se toma el sentido en una línea más deleuziana: "El sentido es pues una noción compleja: siempre hay una pluralidad de sentidos, una constelación, un conjunto de sucesiones, pero también de coexistencias, que hace de la interpretación un arte. Cualquier subyugación, cualquier dominación equivale a una nueva interpretación" (Deleuze, 2013, pp. 10-11).

\footnotetext{
3. Para no dejar lugar a dudas de la manera en que se quiere asumir el término interpretación vale la pena convocar un par de argumentos que permiten seguir clarificándolo: "se plantea la unidad problemática de la crítica y la ontología. La nueva pregunta instaurada por Nietzsche que sustituye a la vieja pregunta metafísica ¿qué es? - de la cual el ¿qué puedo conocer? kantiano no es más que una variante -, es ¿quién?, ¿quién habla?, es la pregunta por la instancia realmente legisladora, es decir, creadora de interpretaciones, instancia activa capaz de conducir la crítica. El quién no es el hombre, no es el sujeto, sino fuerzas en conflicto que dan lugar a interpretaciones que cobran realidad en el lenguaje. De este modo, el hecho del que parte este modo radical de crítica, la existencia efectiva del lenguaje, es al mismo tiempo el dato de la historicidad radical de una realidad- interpretación y, la crítica, una ontología histórica. La arqueología de Foucault no había tomado otra cosa como objeto de sus análisis histórico-críticos: un discurso entendido como práctica que, al entrar en relaciones estratégicas con otras prácticas, se convierte en lugar de articulación de <"lo que pensamos, decimos y hacemos" y, en suma, de constitución histórica de lo real. La pregunta por las condiciones de posibilidad del lenguaje, entendida al modo nietzscheano como la cuestión ¿quién habla?, es la pregunta genealógica por las condiciones de emergencia y procedencia -condiciones simplemente históricas sin duda de una realidad histórica y eventual. Es la nueva pregunta de la crítica, la cuestión antimetafísica por
} 
Entonces, no se encontrará el sentido de algo si no sabemos cuál es la fuerza que se apodera de ello, que lo explota o se expresa en él. En esta medida, a la dualidad metafísica de la apariencia y de la esencia se oponen las fuerzas como expresión de la apropiación, dominación o conducción de una porción de la realidad o bien de los decires, sentires y pensares que pueden configurarse en el escenario múltiple y relacional de la subjetividad. La percepción misma, dice Deleuze (2013), en sus diversos aspectos, es una expresión de fuerzas que se apropian de la naturaleza; la historia de una cosa es la sucesión de fuerzas que se apoderan de ella. En este plano el sentido es pluralista, donde una cosa, una relación, una conducta, una práctica tiene tantos sentidos como fuerzas capaces de apoderarse de ellas. "Pero la propia cosa no es neutra, y se halla más o menos en afinidad con la fuerza que actualmente la posee” (Deleuze, 2013, p. 13). La pretensión está en ubicar la actualidad de las fuerzas que, en medio de las gramáticas como juego de combinación de reglas constitutivas de sentido, operan en los procesos de subjetivación. La Figura 1 permite recrear la propuesta que se viene planteando.

Figura 1. Modelo analítico

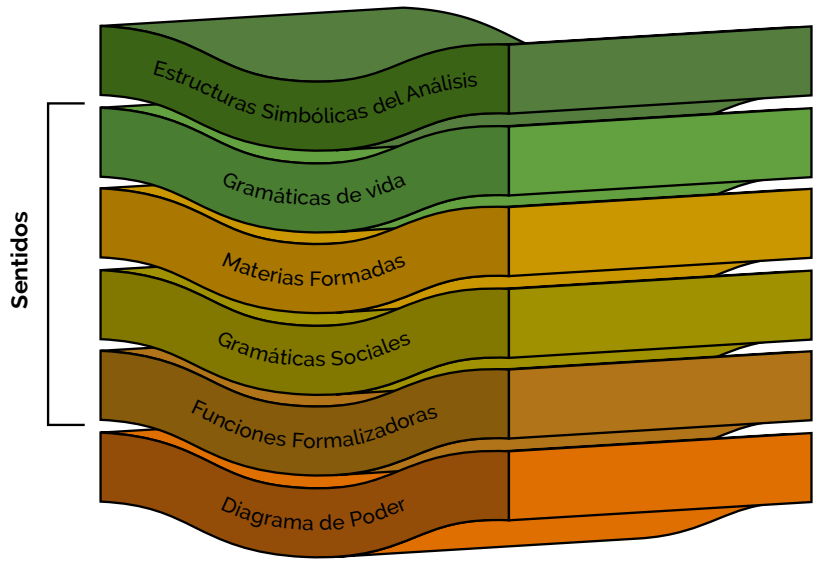

Fuente: elaboración propia

una realidad disuelta en el flujo del devenir de interpretaciones" (De la Higuera, 2003, pp. 23-24. Negrita agregada). De esta manera, quiere recalcarse que la interpretación no es un concepto que solo se puede usar desde las miradas hermenéuticas tradicionales, sino que es la posibilidad de entender también las tensiones entre relaciones de poder que dan lugar a juegos de reglas que unen en ciertos dominios históricos y sociales las palabras y las cosas; así, la interpretación emerge como un pliegue (Deleuze, 2007) temporal de lo heteróclito (Foucault, 1981) en esa medida siempre de modo contingente. 
De esta suerte, la figura en estructura de capas quiere mostrar las categorías que se han venido discutiendo; estas se recrean en un diseño analítico con un interés de mayor claridad, pues se entiende que las realidades no operan necesariamente bajo esta forma escalar y más bien son complejas e interrelacionadas. Ahora bien, se asume una lógica de producción de lo social desde un marco de relaciones de fuerza fluidas que se irán codificando en distintos procesos, el de las funciones formalizadas que da lugar a las gramáticas sociales, sobre el cual emergen desde procesos de subjetivación más específicos las materias formadas en las cuales toman forma las gramáticas de vida. Y, es en estos cuatro últimos estratos en los cuales toman formas los sentidos, unos sentidos que hacen referencia a las fuerzas que se apropian de los sistemas de reglas de juego que dan vida a diversas formas de lo social y distintos modos de subjetivación. De tal modo, para leer estos procesos de manera más fina, es que se usan las técnicas de tratamiento de datos del AEC, como se indica a continuación.

\section{Algunas pistas sobre procedimiento analítico}

De este modo, el MAEC se ubica dentro de las herramientas cualitativas de la investigación y su objetivo es encontrar los esquemas fundamentales de funcionamiento y los principios de ordenamiento del mundo en que se instalan los actores sociales (Suárez, 2008). Este diseño específico de análisis de la información no se puede confundir con análisis del discurso o análisis de textos, estos son los contenedores, modos de expresión o manifestaciones y al MAEC le interesan los contenidos (Hiernaux, 2008). Con lo que son estos contenidos su objeto, de este modo los sentidos, manera de ver las cosas o para este caso los elementos que se analizan son los sentidos y lógicas en que emergen y toman forma las prácticas en tanto que formas de pensar, sentir, decir y hacer que operan en los mundos de las subjetividades. No se puede olvidar entonces que, cuando se habla de prácticas, también se hace alusión a su régimen aglutinador a su gramática, lo que no deja de sugerir un sistema de reglas de juego. De tal modo, se quieren visibilizar gramáticas sociales y gramáticas de vida que se convierten en referencias comunes para el abordaje de los procesos de subjetivación.

El MAEC es, entonces, una estrategia que funciona analizando pequeños pasajes de documentos o fragmentos de decires, emergentes del corpus, que concentren tensiones fundamentales. Estos se llamarán “unidades mínimas de sentido” que organizan la lectura de lo social y del sí, conceptualizadas en clave de este ensamblaje bajo las categorías de gramáticas sociales y gramáticas de vida, que no hacen una lectura fenomenológica o hermenéutica, sino que permiten entender los focos de experiencia (Foucault, 2009), asumidos como la relación 
entre campos de saber, relaciones de poder y las subjetividades posibles que pueden emerger dependiendo de las características de la relación.

Los principios de la descripción estructural, según lo plantea Hiernaux (2008), son la disyunción, la asociación, la isotopía y la condensación descriptiva. En este contexto, decir disyunción contradefinición o distinción (que se gráfica: / ), es referir aquello que permite al interior de un mismo género (totalidad) identificar ciertas cosas como existentes y específicas, unas en relación con otras; es decir, dos términos opuestos entre sí que constituyen una totalidad donde A+$\mathrm{B}=\mathrm{T} \rightarrow$ caliente/frio=Temperatura (Hiernaux, 2008; Suárez, 2008).

Otro de los principios básicos del MAEC es el de asociación o conjunción (que se gráfica: l), el cual, según Hiernaux (2008), pone los elementos ya identificados por las disyunciones, en relación con otros elementos, salidos a su vez de otras disyunciones, formando así la red y los atributos de todos ellos. Posterior a estos puntos, se convoca la isotopía como un tercer principio básico, que pone de manifiesto que el orden de las estructuras que transitan por el material no responden al orden narrativo del mismo.

De tal modo que aquellas "informaciones acerca de un conjunto de unidades de sentido que se articulan entre ellas, que forman "un lugar estructural común" se pueden hallar dispersas en diversos lugares del material" (Hiernaux, 2008, p. 95). El cuarto punto, en tanto que principio básico que se trae a colación, es la condensación descriptiva. Este elemento alude a la diversidad de posibilidades con que cuenta el lenguaje, lo que puede hacer que en un mismo corpus puedan encontrarse múltiples formas de aludir al mismo sentido, el caso de los sinónimos, metáforas, ejemplos, entre otros.

De forma seguida, y sin ser exhaustivos por cuestiones de espacios, estas notaciones se ponen en uso en estructuras simbólicas como las siguientes: estructura paralela, estructura en abanico, estructura cruzada. En primer lugar se despliega una estructura paralela, así, por la lógica de la estructura se agregan dos tipos de códigos disyuntivos que completan las posibilidades del grafo, el código calificativo y el código objeto, del tal modo que una vez instaurados los objetos sobre los cuales se establece la oposición, los códigos calificativos son los que le atribuyen cualidades específicas al código objeto (Suárez, 2008), que en el contexto analizado se le asocian a cada miembro de la disyunción. Usualmente cada polo termina cargado con una valoración cultural positiva (+) o negativa (-) (Saldarriaga, 2008); en ello la herramienta de valorización permite comprender mejor el principio de movilización afectiva y de jerarquización del mundo en que puedan vivir los actores (Suárez, 2008) procede con el siguiente esquema, (Suárez, 2008, p.124): “Un artista? Un hombre que lo sabe todo sin darse cuenta. ¿Un filósofo? Un hombre que no sabe nada, pero que se da cuenta”. En este caso el código objeto sería "un artista / un filósofo", y los códigos calificativos serían "sabe todo / no 
sabe nada” y "no se da cuenta / se da cuenta”, de donde obtenemos la siguiente estructura:

Figura 2. Estructura paralela

$\begin{array}{cccc}\text { Código objeto: } & \text { un artista } & / & \text { un filósofo } \\ \text { Código calificativo 1: } & \text { sabe todo } & / & \text { no sabe nada } \\ \text { Código calificativo 2: } & \text { no se da cuenta } & / & \text { se da cuenta }\end{array}$

Fuente: Suárez, 2008. p. 124.

Usando la valoración (Suárez, 2008): “hace unos años, una dirigente social en Bolivia fue apresada y para su liberación sus simpatizantes manejaron el siguiente slogan: "Wilma Plata, el gobierno fascista la encarcela. El pueblo explotado la libera" (p. 129).

Figura 3. Estructura paralela con valoración

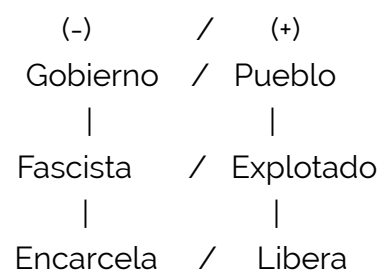

Fuente: Suárez, 2008, p. 129

De otra parte, la estructura en abanico brinda posibilidades cuando en un material se encuentran más de dos elementos que pertenecen a una misma familia, es decir, que no se les puede oponer pues no son de la misma naturaleza. En tal situación se acude a los códigos que los califican y se da lugar a una estructura secuencial, donde paulatinamente los códigos se van organizando en ramificaciones más específicas y forman nuevas totalidades, así hasta concluir con todos los elementos que muestra el material (Suárez, 2008). Para esta estructura se invoca entonces un ejemplo que propone Suárez (2008) retoma un pasaje de una canción de Silvio Rodríguez, Sueño con serpientes, en la cual cita a Bertolt Brecht: "Hay hombres que luchan un día, y son buenos; hay otros que luchan un año, y son mejores; hay quienes luchan muchos años, y son muy buenos; pero hay los 
que luchan toda la vida, esos son los imprescindibles” (Suárez, 2008, p. 131). En este texto tenemos cuatro tipos de hombres, y cada uno tiene su calificativo. La estructura es necesariamente en abanico, pues se organiza del tipo de hombre "bueno" al "imprescindible” con una secuencia paulatina y jerárquica:

Figura 4. Estructura en abanico

Totalidad calf: "tiempo de lucha"

Calf 1:

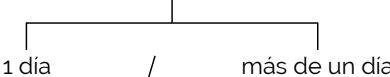

Totalidad Obj.:

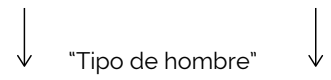

Obj. 1:

bueno $\quad$ / más que bueno

$(-)$

$(+)$

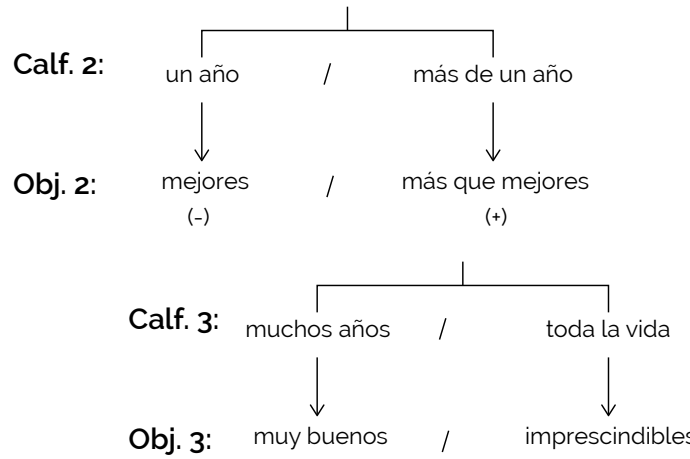

$(-)$

$(+)$

Fuente: Suárez, 2008, p. 131

En tercer lugar, está la alternativa de la estructura cruzada; se usa en el caso de materiales con objetos que simultáneamente tienen el mismo atributo. De este modo, dos códigos disyuntivos calificadores que se cruzan y forman así una estrella con cuatro posibilidades, en las cuales los cuatro códigos-objeto aparecen cada uno con dos calificativos a la vez. En ciertos materiales se pueden conformar estructuras cruzadas con casillas vacías, donde los textos no digan explícitamente qué término corresponde a determinado cruzamiento ${ }^{4}$. El ejemplo sería el siguiente (Saldarriaga, 2003, p. 139):

4. "Esta situación tendrá importantes consecuencias analíticas, pues estaríamos frente a lo que podría decirse explícitamente en el discurso pero, por situaciones específicas, no se verbaliza” (Suárez, 2008, p. 135). 
Figura 5. Estructura cruzada

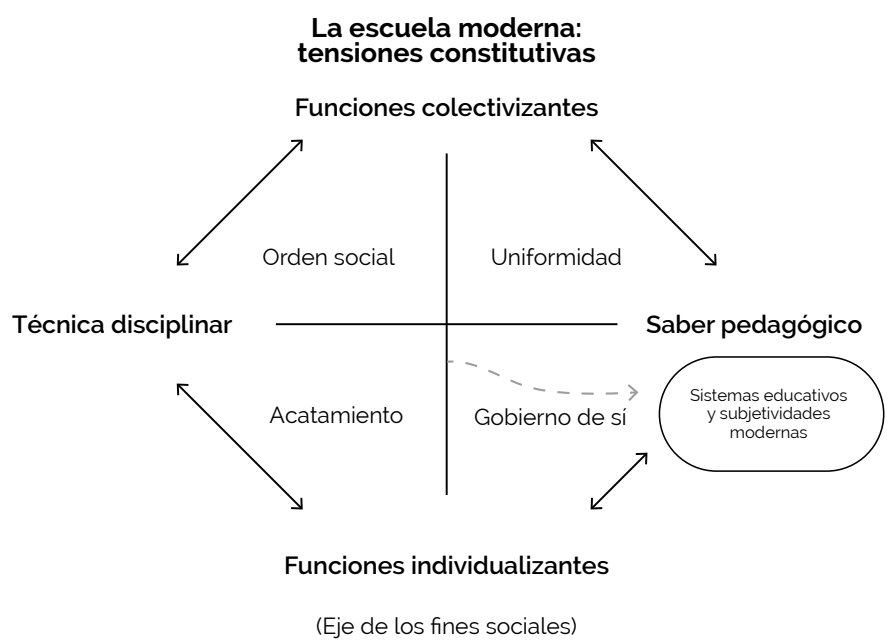

Fuente: Saldarriaga, 2003, p.139

Hay que anotar que la línea punteada retoma una propuesta que plantea Oscar Saldarriaga (2003) desde un lugar de marcado énfasis post-estructuralista. Así, la línea derivada (flecha) hace visibles las líneas de fuga que pueden emerger de las tensiones que se generan en medio de las gramáticas en las que la subjetividad es producida, se-constituye.

\section{Conclusiones}

Se entiende pues que "lo social es complejo y poliédrico y lo que hace cada práctica de investigación es revelar o resaltar, desvelar y polarizar las diferentes dimensiones del fenómeno social investigado" (Conde, 1999, p. 97), por lo que el definir un topos enunciativo como el del post-estructuralismo ya indica una forma muy particular de aproximarse a las realidades y de hacerlas inteligibles en términos analíticos. Con ello, esta propuesta se centra en un análisis que quiere comprender la manera en que se dinamizan los modos de vida socio-históricos desde una mirada de procesos situados, cruzados por la conjunción de campos de saber, relaciones de poder y formas de subjetividad. Leído desde las prácticas sociales que regulan los modos de ser y hacer de las subjetividades, abordándolos tanto en sus gramáticas sociales como campo amplio de posibilidades, que se abren en medio de los diferentes escenarios sociales, al momento que, de sus 
gramáticas de vida, como las apropiaciones o incorporaciones que se dan debido a la regularidad y significatividad de las prácticas por parte de las subjetividades.

En este escenario la conjunción de las gramáticas sociales, las gramáticas de vida y los elementos del MAEC para el tratamiento de los datos, se convierten en una posibilidad muy interesante para el abordaje de objetos de investigación, desde el trabajo con fuentes primarias y fuentes secundarias. Así, pueden elaborarse estructuras simbólicas, teniendo como punto de partida los datos, que van dando lugar a la inteligibilidad de las prácticas y su régimen aglutinador en diferentes espacios sociales.

Esto permite entender de qué manera se configuran ciertos discursos como lugar de verdad en relación con estrategias de poder que los validan, produciendo en lo local las formas que los sujetos asumen como lo verdadero, al momento que pueden permitir dar cuenta de las apropiaciones que hacen que una subjetividad se asuma a sí misma en unas ciertas posiciones y bajo la dinámica de fuerzas de unos ciertos sentidos, produciendo una experiencia de sí. Ello, tejido con un juego de interpretaciones que invocan elementos teóricos, como caja de herramientas, para la compresión de los sentidos, y reclaman la labor de inferencias y desarrollos permanentes por parte del investigador para complementar los análisis que visibilicen las dinámicas sociales y los procesos de subjetivación según sea el caso.

\section{Referencias}

Álvarez, A. (2014). Introducción al post-estructuralismo. Componente de fundamentación en lógicas, enfoque y metodología de producción de conocimiento en ciencias sociales: perspectiva pos-estructuralista. Manizales, Colombia: Centro Internacional de Educación y Desarrollo Humano, CINDE.

Castro-Gómez, S. (2010). Historia de la gubernamentalidad: Razón de Estado, liberalismo y neoliberalismo en Michel Foucault. Bogotá, Colombia: Siglo del Hombre Editores.

Conde, F. (1999). Proceso e instancias de formación-formalización de la multidimensionalidad de lo real: procesos de institucionalización / reificación social en la praxis de la investigación social. En J. Delgado y J. Gutiérrez (Eds.). Métodos y técnicas cualitativas de investigación en ciencias sociales (pp. 97-119). Madrid, España: Editorial Síntesis. 
Creswell, J. W. (2007). Qualitative Inquiry and Research Design: Choosing Among Five Approaches. Los Ángeles, Estados Unidos: Sage.

De la Higuera, J. (2003). Estudio preliminar. En M. Foucault. Sobre la ilustración (pp. 9-67). Madrid, España: Tecnos.

Deleuze, G. (1987). Foucault. Barcelona, España: Paidós.

Deleuze, G. (1995). Conversaciones 1972-1990. Valencia, España: Pre-Textos.

Deleuze, G. (2013). Nietzsche y la filosofía. Barcelona, España: Anagrama.

Deleuze, G. (2014). El poder: curso sobre Foucault. Buenos Aires, Argentina: Cactus.

Foucault, M. (1976). La arqueología del saber. Buenos Aires, Argentina: Siglo XXI.

Foucault, M. (1981). Las palabras y las cosas. Bogotá, Colombia: Siglo XXI.

Foucault, M. (1985). La imposible prisión. Barcelona, España: Anagrama.

Foucault, M. (1988). El sujeto y el poder. En H. Dreyfus; P. Rabinow (Eds.). Michel Foucault: Más allá del estructuralismo y la hermenéutica (pp. 227-244). México, D. F.: Universidad Nacional Autónoma de México.

Foucault, M. (1994). Microfísica del poder. Barcelona, España: Planeta-Agostini.

Foucault, M. (1999a). Obras esenciales. Entre filosofía y literatura. Barcelona, España: Paidós.

Foucault, M. (1999b). Obras esenciales. Estética, ética y hermenéutica. Barcelona, España: Paidós.

Foucault, M. (2002). Historia de la sexualidad. La voluntad de saber. Buenos Aires, Argentina: Siglo XXI.

Foucault, M. (2003a). El yo minimalista y otras conversaciones. Buenos Aires, Argentina: La Marca. 
Foucault, M. (2003b). Historia de la sexualidad. El uso de los placeres. Buenos Aires, Argentina: Siglo XXI.

Foucault, M. (2003c). Sobre la ilustración. Madrid, España: Tecnos.

Foucault, M. (2006a). Defender la sociedad. México: Fondo de Cultura Económica.

Foucault, M. (2006b). Seguridad, territorio y población. Buenos Aires, Argentina: Fondo de Cultura Económica.

Foucault, M. (2008). El nacimiento de la biopolítica. Buenos Aires, Argentina: Fondo de Cultura Económica.

Foucault, M. (2009). El gobierno de sí y de los otros. Buenos Aires, Argentina: Fondo de Cultura Económica.

Foucault, M. (2013). Michel Foucault la inquietud por la verdad: escritos sobre la sexualidad y el sujeto. Buenos Aires, Argentina: Siglo XXI.

Hernández-Sampieri, R.; Fernández, C.; Baptista, P. (2010). Metodología de la investigación. México, D. F.: McGraw Hill.

Herrera, J. D. (2010). La comprensión de lo social. Bogotá, Colombia: Centro Internacional de Educación y Desarrollo Humano, CINDE.

Herrera, J. D. (2013). Pensar la educación, hacer investigación. Bogotá, Colombia: Universidad de La Salle.

Hiernaux, J. (2008). Análisis estructural de contenido y de modelos culturales. Aplicación a materiales voluminosos. En H. Suárez (Ed.). El sentido y el método: sociología de la cultura y análisis de contenido (pp. 67-117). México, D. F.: El colegio de Michoacán.

Hiernaux, J.; Ganty, A. (1977). Sociologie des groupes chrétiens contemporains, systéms symboliques, insertion sociale et mobilization affective. Lovaina la Nueva, Bélgica: AbeBooks

Jaramillo, O. (2013). Una reflexión sobre el método arco-genealógico foucaultiano. Textos $\mathcal{E}^{\circ}$ sentidos, 8, 109-137. https://biblioteca.ucp.edu.co/ojs/index. $\mathrm{php} /$ textosysentidos/article/view/822/782 [Consultado el 11 de julio de 2019]. 
Jaramillo, O. (2018). Gramáticas sociales en los márgenes: formas de gobierno de la vida en el barrio Las Brisas de la ciudad de Pereira. Virajes, 20, 149175. http://vip.ucaldas.edu.co/virajes/downloads/Virajes20(1)_8.pdf [Consultado el 11 de julio de 2019].

Leonard, J. (1985). El historiador y el filósofo. En: M. Foucault. La imposible prisión. Debate con Michel Foucault (pp. 5-36). Barcelona, España: Anagrama.

Morey, M. (1983). Lectura de Foucault. Madrid, España: Taurus Ediciones.

Muñoz, A.; Jaramillo, O. (2019) Constitución-producción de subjetividades a partir de prácticas de sí contemporáneas de un grupo de docentes vinculados al sindicato del magisterio vallecaucano. En A. Redondo, I.; Jaramillo, O. (Eds.). Comprensiones de lo social en clave del desarrollo humano (pp. 9-34). Pereira, Colombia: Universidad Libre.

Sáenz, J. (2014). Artes de vida, gobierno y contraconductas en las prácticas de sí. Bogotá, Colombia: Universidad CES.

Saldarriaga, O. (2003). Del oficio de maestro: Prácticas y teorías de la pedagogía moderna en Colombia. Bogotá, Colombia: Magisterio.

Saldarriaga, O. (2008). Lineamientos curriculares en ciencias sociales. En H. Suárez (Ed.). El sentido y el método: sociología de la cultura y análisis de contenido (pp. 25-50). México, D. F.: Colegio de Michoacán y Universidad Nacional Autónoma de México.

Suárez, H. (2008). El sentido y el método: sociología de la cultura y análisis de contenido. México, D. F.: El colegio de Michoacán.

Trombadori, D. (2010). Conversaciones con Foucault: Pensamientos, obras, omisiones del último maître-à-penser. Buenos Aires, Argentina: Amorrortu.

Veyne, P. (1984). Cómo se escribe la historia. Foucault revoluciona la historia. Madrid, España: Alianza editorial. 
